\title{
Tumores del estroma gastrointestinal (G IST), un particular tipo de neoplasia
}

\author{
Pablo Bórquez Ma, Rodrigo N eveu C. \\ Gastrointestinal stromal tumors. \\ An update
}

Gastrointestinal stromal tumors (GIS) are a heterogeneous group of tumors that express CD 117 molecule in their surface. They may behave as benign tumors or be highly aggressive. A better survival of patients with these tumors has been achieved using the new molecular therapies such as imatinib mesylate, sunitinib and others. This review analyzes the prognostic factors of these tumors, their clinical features and the criteria for malignant behavior. The value of therapeutic alternatives such as radiotherapy, chemotherapy and the new molecular therapies are also discussed (Rev Méd Chile 2008; 136: 921-9).

(Key words: Gastrointestinal stromal tumors; Proein-tyrosine kinases; Proto-oncogene proteins)

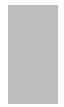

Recibido el 23 de enero, 2007. Aceptado el 28 de septiembre, 2007.
Instituto Nacional del Cáncer
aResidente de Cirugía Oncológica

L

as primeras descripciones de tumores mesenquimáticos del tracto gastrointestinal datan de la década del $40^{1}$. Durante años fueron clasificados como tumores derivados de células musculares lisas basados en el parecido histológico con estas células, así fueron llamados leiomiomas bizarros, leiomiomas celulares y leiomiosarcomas $^{2}$.

Con el advenimiento de la microscopia electrónica se demostró que sólo algunos de estos tumores mostraban evidencias ultraestructurales de presentar diferenciación muscular lisa. La caracterización inmunohistoquímica ha permitido conocer los distintos tipos de diferenciación que

Correspondencia a: Pablo Bórquez. Av. Profesor Zañartu 1010, Independencia, Santiago. Fax: 02- 4484153. E mail: tellomora@yahoo.es presentan estos tumores. En 1984, Herrera et $\mathrm{al}^{3}$ describieron una diferenciación neuronal autonómica en algunos subtipos de este tumor que desde 1983, gracias al trabajo de Manzur y Clark ${ }^{4}$, se habían denominado genéricamente como "tumores estromales". Pese a los avances, aun en la década de los 80 persistían las dudas dado que estos tumores poseen diferenciación miogénica, neural, pero también pueden presentar diferenciación mixta o sencillamente no presentar diferenciación específica. Otro hito en la historia de esta enfermedad lo marcó Hirota et al. que en 1998 descubre mutaciones funcionantes en el protooncogén c-kit de los GIST, lo que permitió a la larga una clasificación adecuada de los tumores, así como el desarrollo de una novedosa terapia molecular dirigida contra la tirosina kinasa ${ }^{5}$.

Se ha sugerido que los GIST se originan de las células intersticiales de Cajal o desde células precursoras primitivas que se diferencian hacia 
células intersticiales de Cajal y hacia células fenotípicamente musculares lisas ${ }^{6}$. Las células intersticiales de Cajal se encuentran intercaladas entre los nervios autonómicos y las células musculares lisas, su principal función es generar el ritmo autónomo de contracciones involucradas en la digestión y peristaltismo, de modo que son también llamadas células marcapaso del tracto gastrointestinal.

Los GIST constituyen un grupo relativamente poco frecuente de neoplasias, representa menos del $1 \%$ de los tumores primarios del tracto gastrointestinal y tiene una incidencia aproximada de entre 10 y 20 por millón ${ }^{7}$, la cual parece ser constante en las distintas áreas geográficas y etnias. Tres trabajos recientes ${ }^{8-10}$, en el norte de Europa, basados en estudios poblacionales y usando similares criterios diagnóstico informan la incidencia anual de GIST en 14,5 por millón en Suecia, 11 por millón en Islandia y 12,7 por millón en Holanda, en estas poblaciones aproximadamente un $10 \%$ de los casos son diagnosticados por endoscopía o imágenes abdominales o cirugía por otras condiciones. Aparecen predominantemente en edades medias y ancianos (60 años en promedio). No habrían diferencias entre sexos, excepto en pacientes portadores de la triada de Carney ${ }^{11}$ (GIST gástrico maligno, condroma pulmonar y paraganglioma extra adrenal funcionante), que ocurre más frecuentemente en mujeres. No tienen factores de riesgo conocidos, sin embargo, parece haber una influencia genética que se aprecia en reportes de GIST familiares y otros asociados a la enfermedad de von Recklinghausen ${ }^{12}$.

Son tumores muy heterogéneos que varían en tamaño, morfología y conducta biológica, son un continuo de neoplasias con potencial maligno incierto comportándose virtualmente como tumores benignos hasta cánceres muy agresivos, muchas veces metastásicos al diagnóstico. El grupo de tumores agresivos representa entre un 20 y un $45 \%$ de todos los $\operatorname{GIST}^{7,8}$, y tendría una incidencia de 5 por millón de habitantes. En Chile no conocemos su incidencia y hay algunos reportes 0 series de casos publicados esporádicamente en la literatura local que permite hacerse una idea de lo infrecuentes que son en nuestra población ${ }^{13-17}$.

El objetivo de este trabajo es presentar una revisión actualizada de este interesante y poco frecuente grupo de patologías que, gracias a los avances en biología molecular y marcadores, ha podido recientemente ser caracterizada.

Definición actual. El protooncogen c-kit, localizado en el brazo largo del cromosoma 4, codifica el receptor transmembrana de tirosina kinasa tipo III. El producto de este gen, la proteína kit tiene un dominio extracelular que es un receptor y un dominio intracelular ligado a una tirosina kinasa. El ligando del receptor es un factor de crecimiento de células precursoras, una vez ligado produce dimerización del receptor, gatillando la fosforilación del dominio kinasa, esto genera una cascada de señales desde el citoplasma al núcleo donde afecta el comportamiento de la célula en su proliferación, diferenciación, adhesión y apoptosis. La expresión de kit (o CD 117 según la terminología estandarizada de antígenos leucocitarios) es extremadamente importante para algunas líneas celulares normales como células precursoras hematopoyéticas, mastocitos, células germinales (espermatogonias y espermátidas), células de Leydig, melanocitos, algunas células epiteliales (del estrato basal de la piel y epiteliales de mama) y también para las células intersticiales de Cajal, células tumorales de Leucemia Mieloide Crónica y GIST ${ }^{18-20}$.

La expresión de CD 117 ha emergido como el marcador para discriminar el GIST de otros tumores mesenquimáticos gastrointestinales, así su expresión ha pasado a ser casi sinónimo de GIST $^{7}$. Sin embargo, pese a ser altamente sensible es menos específico, ya que puede encontrarse expresado en células tumorales de Sarcoma de Ewing, angiosarcoma, melanoma, cáncer pulmonar de células pequeñas, carcinoma ovárico, linfoma, leucemia, seminoma y neuroblastoma ${ }^{16}$. La caracterización inmunohistoquímica de los GIST incluye además del CD 117 al CD34 (marca positividad alrededor de los ganglios del plexo mioentérico de Auerbach en el tracto gastrointestinal). Ambos, CD117 y CD34 son positivos en GIST en valores superiores al $90 \%$. Otros marcadores de inmunohistoquímica sirven para determinar el tipo de diferenciación del tumor, así la inmunoreacción focal de actina de músculo liso (SMA) es diagnóstica de diferenciación muscular y otros, como el S-100 lo es de diferenciación neural ${ }^{21}$. Los GIST frecuentemente son vimentina positivo y usualmente desmina negativo. Todos ellos son 
importantes en el diagnóstico diferencial de otros tumores que pueden expresar CD 117 como melanoma y plasmocitoma ${ }^{22}$.

Las secuencias de ADN para c-kit demuestran una alta frecuencia de mutaciones que determinan la activación de la tirosina kinasa en ausencia de la estimulación por su ligando fisiológico presentando de este modo una proliferación celular aberrante y resistencia a la apoptosis. La mutación más frecuente identificada está en el exón 11 (dominio yuxtamembrana), otras mutaciones en el gen c-kit incluyen defectos en el exón 9, 13 y 17, pero son mucho menos frecuentes ${ }^{23}$. Existe un grupo entre un 5 y un $15 \%$ de los GIST que no expresan el CD 117, en estos pacientes hay mutaciones del receptor alfa del factor de crecimiento derivado de plaquetas o PDGFRA, otra tirosina kinasa, pero este marcador no logra caracterizar a la mayoría de los GIST. Esta mutación provee de un mecanismo oncogénico alternativo a estos tumores ${ }^{24,25}$.

Hay una asociación entre GIST y Neurofibromatosis tipo 1 (NF1). Los pacientes con NF1 son portadores de un GIST en el 7\% de los casos, sin embargo el mecanismo por el cual esto ocurre es desconocido $^{26}$. Se han intentado reconocer mutaciones en kit y PDGFRA de pacientes con NF1 sin éxito. Sin embargo, en células tumorales de GIST ha sido demostrada que la inactivación somática del gen supresor de tumor NF1 debido a una mutación ${ }^{27}$.

Características clínicas: El GIST abarca un amplio espectro de cuadros que va desde tumores benignos hasta tumores altamente agresivos. Los tumores pequeños pueden ser asintomáticos $\mathrm{y}$ encontrarse como un hallazgo endoscópico, laparoscópico o por imágenes. Pueden producir dolor abdominal mal caracterizado, saciedad precoz, ictericia, disfagia, fiebre y otros, aunque la forma de presentación más frecuente es como una hemorragia gastrointestinal hacia la cavidad abdominal produciendo abdomen agudo y anemia 0 hacia el lumen del tubo digestivo produciendo hematemesis, melena y anemia ${ }^{28}$.

El GIST puede producirse en cualquier punto del tracto gastrointestinal desde el esófago hasta el ano, sin embargo el estómago (39 a 60\%) y el intestino delgado (30 a 42\%) son los sitios más frecuentes de localización de estos tumores. En colon, recto, apéndice, esófago, mesenterio, retroperitoneo y otros órganos intraabdominales son ubicaciones inhabituales del GIST 7,29 .

Los pacientes portadores de GIST tienen un alto riesgo de presentar segundos tumores primarios del tracto gastrointestinal, lo que ha sido reportado en pequeñas series y reportes de casos. Su incidencia no está claramente definida, sin embargo parece ser tan alta como $14 \%$. Los segundos tumores primarios más frecuentes en pacientes portadores de GIST son carcinoma gástrico, carcinoma de colon y linfoma gástri$\mathrm{Co}^{30}$.

El tipo agresivo del GIST se presenta con compromiso local en cavidad abdominal (en peritoneo, mesenterio, omento) o como compromiso a distancia con metástasis hepáticas. La metástasis extraabdominal es rara. La sintomatología del GIST metastásico es inespecífica, así como en otros tumores metastásicos sus manifestaciones clínicas más frecuentes son baja de peso y dolor abdominal mal precisado ${ }^{22}$. Puede implantarse en cicatrices quirúrgicas y en trayectos de agujas por paracentesis. El compromiso linfonodal es muy poco frecuente ${ }^{28}$. Son lesiones que frecuentemente se diagnostican por TAC o una endoscopía sugerente. La asociación de endoscopía y ecografía (endosonografía) puede ayudar al diagnóstico de certeza, así los GIST son infrecuentes en esófago, por lo tanto una lesión esofágica subepitelial hipoecogénica de la $2^{\mathrm{a}}$ capa (muscular de la mucosa) 0 de la $4^{\text {a }}$ (capa muscular) es más probable que se deba a un leiomioma, en cambio la misma lesión en el estómago como primera posibilidad corresponde a un GIST. La combinación de la endosonografía con PAAF (punción aspirativa con aguja fina) puede permitir obtener una muestra adecuada para realizar un diagnóstico de GIST. ${ }^{31}$

Morfología y criterios de malignidad: Macroscópicamente van de unos pocos $\mathrm{mm}$ hasta varios $\mathrm{cm}$ (hay tumores de 30 y más $\mathrm{cm}$ ), son lesiones difusas, encapsuladas o multinodulares, fibrosos al corte con áreas de hemorragia, degeneración quística y necrosis central. Habitualmente son tumores intramurales, usualmente comprometen la submucosa y la muscular propia. Los tumores grandes pueden colgar hacia el lumen o hacia la cavidad peritoneal. Sin embargo, su apariencia 
macroscópica, salvo en el compromiso metastásico evidente, no orienta hacia un fenotipo agresivo.

Microscópicamente pueden ser tipo células fusadas en $70 \%$ de los casos, epitelioide en un $20 \%$, y el resto es del tipo mixto. Un pequeño porcentaje, menor a $5 \%$, tiene un marcado pleomorfismo nuclear.

A veces es difícil predecir cuáles tumores tendrán un comportamiento agresivo, sin embargo existen factores clínico-patológicos o citogenéticos que se consideran predictores de malignidad en GIST.

I. Factores pronóstico clínicos y patológicos.

A. Presencia de metástasis. La presencia de metástasis peritoneales y hepática al diagnóstico es un factor pronóstico adverso asociado con una breve sobrevida ${ }^{32}$.

B. Tamaño tumoral, ubicación del tumor primario y actividad mitótica. Según el tamaño tumoral y el conteo mitótico pueden definirse grupos de distinto riesgo ${ }^{33}$ (Tabla 1).

La ubicación del tumor primario parece ser un factor de riesgo independiente del tamaño tumoral y del conteo mitótico, por ejemplo, el tumor de intestino delgado tiene peor pronóstico, así puede evaluarse cada tumor de manera sitio específica. Según esto se han definido 3 categonías: tumor benigno, maligno y de comportamiento incierto o bajo potencial maligno ${ }^{33}$ (Tabla 2).

C. Tipo epitelioide, el patrón celular es positivo en $1 / 3$ de los tumores gástricos pero tiene mayor potencial maligno si se ubica en intestino. La baja celularidad se considera un factor pronóstico favorable.

D. Invasión de la mucosa es rara de ver en GIST pero se la considera un factor pronóstico adverso. Debe diferenciarse de la ulceración por compromiso isquémico que puede verse en los fenotipos benigno y maligno y no constituye un factor pronóstico ${ }^{34}$.

Tabla 1. Categorías de riesgo de malignidad según tamaño tumoral y conteo mitótico (por histología)

\begin{tabular}{|lcc|}
\hline Categoría de riesgo & $\begin{array}{c}\text { Tamaño tumoral } \\
(\mathrm{cm})\end{array}$ & $\begin{array}{c}\text { Conteo mitótico } \\
\text { (mitosis por campo) }\end{array}$ \\
\hline Muy bajo riesgo & $<2$ & $<5$ \\
Bajo riesgo & $2-5$ & $<5$ \\
Riesgo intermedio & $<5$ & $6-10$ \\
& $5-10$ & $<5$ \\
Alto riesgo & $>5$ & $>5$ \\
& $>10$ & cualquier conteo \\
& cualquier tamaño & $>10$ \\
\hline
\end{tabular}

Tabla 2. Criterios histológicos de malignidad en GIST

\begin{tabular}{|ll|}
\hline Categoría & Criterio \\
\hline GIST benigno & Tumor intestinal $\leq$ de $2 \mathrm{~cm}$ y no más de 5 mitosis \\
& Tumor gástrico de $\leq 5 \mathrm{~cm}$ y no más de 5 mitosis \\
Malignidad & Tumor intestinal $>2 \mathrm{~cm}$ pero $\leq 5 \mathrm{~cm}$ y no más de 5 mitosis \\
Intermedia & Tumor gástrico $>5 \mathrm{~cm}$ pero $\leq 10 \mathrm{~cm}$ y no más de 5 mitosis \\
GIST maligno & Tumor intestinal $>5 \mathrm{~cm}$ o más de 5 mitosis \\
& Tumor gástrico $>10 \mathrm{~cm}$ o más de 5 mitosis \\
\hline
\end{tabular}


II. Marcadores citogenéticos. La predicción del comportamiento biológico en base a parámetros clínicos y patológicos no siempre es posible. Existen distintos parámetros que se perfilan como útiles factores pronóstico a partir de estudios citogenéticos.

A. C-kit y mutaciones del PDGFRA, las mutaciones de ambos son conocidas por ser pasos críticos en la iniciación de eventos oncogénicos, sin embargo, el valor pronóstico y su utilidad terapéutica aún se encuentran en fase de investigación. Se sabe que mutaciones (delecciones) del exón 11 son por ej. predictores independientes de sobrevida libre de enfermedad ${ }^{35}$.

B. Aberraciones cromosómicas, se ven en todos los tipos de GIST independientemente del tipo, por lo que parecen relacionarse con eventos muy precoces en la patogenia del tumor, sin embargo se ven algunos cambios como la pérdida de zonas del cromosoma como 1p, 9q y 9p en fenotipos agresivos o hay más frecuencia de delecciones en los tumores recurrentes que en los primarios. Estos parecen ser eventos más tardíos y jugarían un rol en la progresión tumoral. ${ }^{36}$

C. Actividad de telomerasa. La telomerasa es una enzima que se encuentra reprimida en células somáticas y activa en células germinales y tumorales, así las células sufren acortamiento de la longitud del telómero con las divisiones sucesivas, esto detiene el ciclo celular, sin embargo algunos clones estabilizan sus cromosomas y adquieren la propiedad de crecer indefinidamente. La actividad de telomerasa ha sido detectada en GIST maligno y está ausente en tumores benignos. ${ }^{37}$

\section{TRATAMIENTO}

a) Radioterapia. Es un tumor radioresistente, además como otros sarcomas intraabdominales no pueden ser tratados con radioterapia por la importante morbilidad que se produciría al estar ubicados vecinos a otros órganos, que toleran limitadamente las dosis de radiación a administrar. Se han descrito tratamientos con radioterapia en GIST fijo a pared abdominal o irresecables fijos a otros órganos, pero con malos resultados ${ }^{38}$. La radioterapia no es un estándar de tratamiento adyuvante en GIST, podría tener algún rol en casos seleccionados como paliación.

b) Quimioterapia. La quimioterapia sistémica no ha sido efectiva. Hay algunos estudios con baja tasa de respuesta a combinaciones de doxorrubicina y dacarbacina ${ }^{39}$, dacarbacina más mitomicina c, doxorrubicina y cisplatino ${ }^{40}$ y no hay respuesta a etopósido e ifosfamida ${ }^{41}$.

La falta de respuesta a las drogas usualmente usadas en sarcomas de partes blandas se debería a la frecuente expresión de la glicoproteína $\mathrm{P}$ y proteína a multiresistencia a drogas 1(MDR1) en estos tumores, relacionados con la resistencia a drogas $^{42}$.

c) Rol de la cirugía: La cirugía es el estándar de tratamiento para el GIST no metastásico. Debiera resecarse con criterio oncológico parecido a otros sarcomas intraabdominales, es decir, con un margen libre de tumor y no a través de la pseudocápsula que se produce en la interfase entre tumor y órganos y tejidos adyacentes, sin embargo el criterio para considerar una resección $\mathrm{R}_{0}$ no ha sido precisado. De involucrar órganos adyacentes se recomienda una resección en bloque de modo de obtener el margen oncológico, así puede requerirse una resección anterior baja o una operación de Miles para resecar un tumor rectal o una gastrectomía para tratar un tumor gástrico, debido a la demostrada mejor sobrevida de los pacientes tratados más agresivamente. ${ }^{36,43}$

La ruptura del tumor espontáneamente o en el intraoperatorio se asocia a un alto riesgo de implante peritoneal y debe ser evitado, ya que son tumores frágiles cuando tienen hemorragia, degeneración quística o necrosis intratumoral. Debido a la baja frecuencia de metástasis ganglionares no se recomienda realizar linfadenectomía regional.

d) Mesilato de imatinib y cirugía: El mesilato de imatinib es un derivado 2-fenilaminopirimida administrado por vía oral $\left(\right.$ Glivec $^{\mathrm{R}} \mathrm{Gleevec}^{\mathrm{R}}$ ) que actúa como un inhibidor competitivo del dominio que liga ATP de kit, PDGFRA y otras tirosina kinasas $^{44,45}$. Así el imatinib inhibe la actividad kinasa de la tirosina kinasa evitando su fosforilación y la activación de la cascada de señales intracelular. En estudios preclínicos se demostró 
una inhibición de la proliferación celular y un aumento de la apoptosis dosis dependiente ${ }^{46}$. Tiene una vida media de $16 \mathrm{hr}$ y los efectos adversos descritos durante el tratamiento son hemorragia digestiva por necrosis tumoral, diarrea, edema periorbitario, eczema, disfunción hepática moderada, mialgias y fatiga. Requiere concentraciones séricas de 1umol/lt para lograr un efecto terapéutico, esto se logra con dosis mayores o iguales de $300 \mathrm{mg} /$ día.

Varios estudios demostraron la utilidad de imatinib en GIST metastásico ${ }^{47,48}$. Antes de que se aceptara su indicación en enfermedad metastásica la media de sobrevida de los pacientes era menos de 12 meses, en la era actual se han descrito sobrevidas mayores a 2 años, en base a respuestas parciales ( $59 \%$ vs $13 \%$ sin imatinib). Un estudio europeo comunica un $69 \%$ de respuesta en 36 pacientes ${ }^{49}$, sin embargo, la tasa de respuesta completa es menor a un $5 \%$ de los $\operatorname{casos}^{50}$. Por otra parte hay descrita resistencia primaria y secundaria (adquirida) a la terapia con imatinib. Se han descrito mutaciones en el exon 9, 11 y 17 del dominio kit kinasa. Son estudios citogenéticos realizados en series pequeñas y reportes de casos que por su costo no están disponibles para evaluar la respuesta a la terapia pre-tratamiento, una vez diagnosticada una resistencia podría, en casos seleccionados evaluarse su utilidad o buscar una terapia molecular de $2^{\mathrm{a}}$ línea, no obstante y por un criterio de costo/ beneficio estas mutaciones no tienen un rol en la evalución de una posible resistencia a drogas pre tratamiento. ${ }^{51}$ Recientemente la Administración de Drogas y Alimentos de los Estados Unidos de Norteamérica (FDA) ha aprobado la droga sunitinib maleato como segunda línea para el tratamiento del GIST que ha progresado pese al tratamiento con imatinib o es resistente a él. La sobrevida libre de progresión fue 4 veces mayor en sunitinib vs placebo (27,3 semanas vs 6,4 semanas) ${ }^{52}$ y constituye una alternativa para estos pacientes.

Respecto el imatinib, hay 2 hechos innegables, el primero es que sin duda ha revoluciona- do el tratamiento del GIST metastásico, pero no reemplaza ni puede paliar un inadecuada cirugía inicial $^{53}$, otro hecho importante es que aún no está claro un aumento real de la sobrevida medida a partir de información poblacional debido a los aumentos que ha tenido la incidencia de GIST a partir de su redefinición con el uso de marcadores moleculares ocurrido hace no más de 5 años. ${ }^{54}$

El rol de la cirugía posterior al uso de imatinib (usado como neoadyuvancia) no está claro. En teoría podría facilitar la resección al reducir la vasculatura y el volumen tumoral, pero técnicamente sería más demandante al producirse degeneración mixoide o hialina al interior del tumor. Hay descritos algunos casos de respuesta patológica completa con ausencia de la expresión de CD 117 en tumores metastásicos resecados posterior al uso de imatinib, pero son casos aislados ${ }^{55}$. Pese a esto la utilidad de la resección de metástasis 0 de la enfermedad recurrente no se conoce con exactitud. La mortalidad específica y la sobrevida no fue distinta entre pacientes con resección completa de la recurrencia vs pacientes en quienes se realizó una resección parcial o sólo una biopsia. Podría tener un efecto en pacientes seleccionados, pacientes con tumor bien o moderadamente diferenciado, con breve intervalo libre de enfermedad entre el diagnóstico y la detección de metástasis y la metástasis hepática única resecable, este grupo de pacientes se beneficiaría con cirugía resectiva ${ }^{56}$.

El uso de imatinib como adyuvante de la cirugía en tumores no metastásicos de alto riesgo no cuenta aún con evidencia de buen nivel que la soporte. Hay 2 estudios en curso que intentarán responder esta pregunta ${ }^{57}$. El primero es un estudio en fase II en lesiones de alto riesgo (lesiones grandes, tumores complicados o multifocales en peritoneo y el segundo un ensayo clínico doble ciego aleatorizado en fase III de imatinib vs placebo. Durante 2007-2008 probablemente conoceremos resultados preliminares que apoyen o no su uso como adyuvante de la cirugía en GIST no metastásico. 


\section{REFERENCIAS}

1. Golden T, Stout AP. Smooth muscle tumours of the gastrointestinal tract and retroperitoneal tissues. Surg Ginecol Obstet 1941; 73: 784-810.

2. SтоUт AP. Bizarre smooth muscle tumours of stomach. Cancer 1976; 15: 400-9.

3. Herrera GA, Pinto DM, Grizzie WE, Han SG. Malignant small bowel neoplasm of enteric plexus derivation (plexosarcoma). Light and microscopic study confirm the origin of neoplasm. Dig Dis Sci 1984; 29: 275-84.

4. Manzur MT, Clark HB. Gastric stromal tumours. Reappraisal of histogenesis. Am J Surg Pathol 1983; 7: 507-19.

5. Hirota S, Isozaki K, Moniyama $Y$, Hashimoto K, Nishida T, IsHigmo $S$ ET AL. Gain of function mutation of $\mathrm{c}$ kit in human gastrointestinal stromal tumors. Science 1998; 279: 577-80.

6. Rubin BP, Fietcher JA, Fietcher CD. Molecular insights into the histogenesis and patogenesis of gastrointestinal stromal tumours. Int J Surg Pathol 2000; 8: 5-10.

7. Miettinen M, Lasota J. Gastrointestinal stromal tumours - definition, clinical, histological, inmunohistochemical and molecular genetic features and differential diagnosis. Virchows Arch 2001; 438: 1-12.

8. Nilson B, Bumming P, Meis-Kindblom JM, Oden A, Dortok A, Gustavsson B Et aL. Gastrointestinal stromal tumours: The incidence, prevalence, clinical course and pronostication in the pre imatinib mesylate era. Cancer 2005; 103: 821-9.

9. Goettsch WG, Bos SD, Breekveidt-Postma N, Casparie M, Herings RM, Hogendoorn PC. Incidence of gastrointestinal stromal tumours is understimated: Results of a nation-wide study. Eur J Cancer 2005; 41: 286872.

10. Tryggvason G, Gislason HG, Magnusson MK, Jonasson JG. Gastrointestinal stromal tumors in Iceland, 19902003: The Icelandic GIST study, a population based incidence and patologic risk stratification study. IntJ Cancer 2005; 117: 289-93.

11. CARNEY JA. Gastric stomal sarcoma, pulmonary condroma and extra adrenal paraganglioma (Carney triad): Natural history, adreno cortical component and posible familial occurrence. Mayo Clin Proc 1999; 74: 543-52.

12. Giuly JA, Picard R, Giuly D, Monges B, Nguyen-Cat R. Von Recklinghausen disease and gastrointestinal stromal tumours. Am J Surg 2003; 185: 86-7.

13. Cardemil G, Fernández E, Moyano L, Carreño L, Zepeda G, VARGAS F. Tumor estromal de colon fistulizado a pleura (GIST). Rev Chil Cir 2002; 54: 539-43.

14. Cárdenas R, Tapia A, Riquelme R. Leiomiosarcoma colorrectal. Rev Chil Cir 1997; 49: 701-4.
15. Jensen C, Moyano L,Cúneo A, Abedrapo M, Bocic G, GarRIDo R ET al. GIST de colon. Diagnóstico y factores pronóstico. Rev Chil Cir 2001; 53: 91-4.

16. Carvajal $C$, Iturra $S$, Justiniano $P$, Bustamante $M$, Contreras JE, Lombardi J ET aL. Tumores estromales gastrointestinales duodenales. Rev Méd Chile 2006; 134: 481-4.

17. Braghetto I, Parada F, Cardemil G, Csendes A, FernánDEZ E, Korn O ET AL. Tumores gastrointestinales estromales (GIST): Experiencia del Servicio de Cirugía del Hospital Clínico de la Universidad de Chile entre 1999 y 2005. Rev Méd Chile 2007; 135: 551-7.

18. Vlagofis H, Worobac AS, Metcalfe DD. The proto oncogen c-kit and c-kit ligand in human disease. J Allergy Clin Inmunol 1997; 100: 435-40.

19. Heinrich MC, Blanke CD, Druker BJ, Coriess CL. Inhibition of kit tyrosine kinase activity: a novel molecular approach to the treatment ok kit positive malignances. J Clin Oncol 2002; 20: 1962-703.

20. Arber DA, Tamayo R, Weiss LM. Paraffin section detection of the $\mathrm{C}$ kit gene product (CD 117) in human tissues: value in the diagnosis of mast cell disorders. Hum Pathol 1998; 28: 498-504.

21. Lu FY, QI JP, WU AP. Clinicopathological and inmunohistochemical analysis of gastrointestinal stromal tumor. World J Gastroenterol 2006; 12: 4161-5.

22. Bucher P, Vimiger P, Egger JF, Buhler L, Morel PH. Management og gastrointestinal stromal tumours: from diagnosis to treatment. Swiss Med WKLY 2004; 134: 145-53.

23. Corless CL, Fietcher JA, Heinrich MC. Biology of gastrointestinal stromal tumors. J Clin Oncol 2004; 22: 3813-25.

24. HeinRich MC, Coriess CL, Demetri GD, Bianke CD, von Mehren M, Joensuu H. Kinase mutations and imatinib response in patients with metastatic gastrointestinal stromal tumor. J Clin Oncol 2003; 21: 4342-9.

25. Heinrich MC, Corless CL, Duensing A, Mc Greevey L, CHEN CJ, JOSEPH N ET AL. PDGFRA activating mutations in gastrointestinal stromal tumors. Science 2003; 299: 708-10.

26. Soljer Me, Rembeck B, Oden A, Samuelsson M, Angewall L Malignant and benign tumors in patients with neurofibromatosis type 1 in a defined Swedish population. Cancer 1997; 79: 2125-31.

27. Maertens O, Prenen H, Debiec-Rychter M, Wozniak A, Sciot R, Pauwels P et al. Molecular patogénesis of múltiple gastrointestinal stromal tumours in NF1 patients. Human Molecular Genetics 2006; 15: 101523.

28. JoEnsuU H. Current perspectives on the epidemiology of gastrointestinal stromal tumours. EJC Supplements 2006; 4 Suppl: 4-9.

29. EINSENBERG BL, JuDSON I. Surgery and imatinib in the management of GIST: emerging approaches to 
adyuvant and neoadyuvant therapy. Ann Surg Oncol 2004; 11: 465-75.

30. Wronski M, Ziarkiewicz-Wroblewska B, Gornicka B, Cebulski W, SLodkowski M, Wasiutynski A eT aL. Synchronous occurrence of gastrointestinal stromal tumourss and other primary gastrointestinal neoplasms. Worl J Gastroenterol 2006; 12: 5360-2.

31. Gómez M, Otero W, Arbelaez V, Rodríguez J. Ecoendoscopia. Indicaciones de la $\mathrm{A}$ a la Z. Rev Col Gastroenterol 2005; 20: 34-64.

32. Dematteo RP, Lewis JJ, Leung D, Medan SS, Woodruff JM, BRENNAN MF. Two hundred gastrointestinal stromal tumors: recurrence patterns and pronostic factors for survival. Ann Surg 2000; 231: 151-8.

33. Fletcher CD, Berman JJ, Corless C, Gorstein F,Lasota J,LONGLEY BJ. Diagnosis of gastrointestinal stromal tumors: a consensus aproach. Int J Surg Pathol 2002; 10: 81-9.

34. Miettinen M, El Rifai W, Sobin Hl, Lasota J. Evaluation of malignancy and pronostic of gastrointestinal stromal tumors: a review. Hum Pathol 2002; 33: 478-83.

35. Singer S, Rubin BP, Lux ML, Chen CJ, Demetri GD, FLETCHER CD. Prognostic value of kit mutation type, mitotic activity and histologic sub type in gastrointestinal stromal tumors. J Clin Oncol 2002; 20: 3898905.

36. Antonescu CR, Sommer G, Sarran L, Tschernyavsky SJ, Riedel E, Woodruff JM et AL. Association of kit exon 9 mutations with non gastric primary site and agressive behaviour: kit mutation análisis and clinical correlates of 120 gastrointestinal stromal tumors. Clin Cancer Res 2003; 9: 3329-37.

37. Sakurai S, Fukayame M, Kaizaki Y, Saito K, Kanazana $\mathrm{K}$, KitamuRa M. Telomerase activity in gastrointestinal stromal tumors. Cancer 1998; 83: 2060-6.

38. Crosby JA, Catton CN, Davis A, Couture J, O 'Suluivan B, KANDEL R. Malignant gastrointestinal stromal tumors of the small intestine:a review of 50 cases from a prospective database. Am Surg Oncol 2001; 8: $50-9$

39. Zalupski M, Metch B, BalcerZak S, Fletcher WS, ChAPMAn R, Bonnet JD. Phase III comparison of doxorrubicin and dacarbazine given by bolus versus infusión in patients with soft tissue sarcomas: a Southwest Oncology Group Study. J Natl Cancer Inst 1991; 83: 926-32.

40. Edmonson J, Marks R, Bucker J. Contrast of response to D-MAP plus sargramostin between patients with advanced malignant gastrointestinal stromal tumors and patients with others advanced leiomyosarcomas. Proc Am Assoc Cancer Res 1999; 18: (abstr 541).

41. Blair SC, Zalupski M, BaKer LH. Ifosfamide and etoposide in the treatment of advanced soft tissue sarcomas. Am J Clin Oncol 1994; 17: 480-4.
42. Plaat Be, Hollema H, Molenaar WM, Torn Broers GH, Pijpe J, Mastik MF et al. Soft tissue leiomyosarcomas and malignant gastrointestinal stromal tumors: differences in clinical outcome and expression of multidrug resistance proteins. J Clin Oncol 2000; 18: 3211-20.

43. Chou FF, Eng HL, Sheen-Cheu SM. Smooth muscle tumours of the gastrointestinal tract:analisis of prognostic factors. Surgery 1996; 119: 171-7.

44. Buchdunger E, Zimmermann J, Mett H, Meyer T, Muler M, Druker BJ. Inhibition of the Abl protein tyrosine kinase in vitro and in vivo by 2-phenylaminopyrimide derivate. Cancer Res 1996; 56: 100-4.

45. Buchdunger E, Cioffi CL, LaW N, Stover D, OhnoJONES S, DRUKER BJ. Abl protein tyrosine kinase inhibitor STI571 inhibits in vitro signal transduction mediated by c-kit and platelet derived growth factor receptorss. J Pharmacol Exp Ther 2000; 295: 139-45.

46. Heinrich MC, Griffith DJ, Durker BJ, Wait CL, OtT KA, ZigLET AJ ET AL. Inhibition of c-kit receptor tyrosine kinase activity by STI571, a selective tyrosine kinase inhibitor. Blood 2000; 96: 925-32.

47. Joensuu H, Roberts PJ, Sarlomo-Rikala M, Andersson LC, Tervahartiala P, Tuveson D. Effect of the tyrosine kinase inhibitor STI571 in a patient with a metastatic gastrointestinal stromal tumor. $\mathrm{N}$ Engl J Med 2001; 344: 1052-6.

48. Van Oosteron AT, Judson I, Verweij J, Stroobants S, Donato di Paola E, Dimitrijevic S. Safety and efficacy of imatinib (STI571) in metastatic gastrointestinal stromal tumors: a phase I study. Lancet 2001; 358: 1421-3.

49. Debiec-Rychter M, Dunez H, Judson I, Wasag B, Veerweij J, Brown M et al. EORTC Soft Tissue and Bone Sarcoma Group. Use of c-kit/PDGFRA mutational analisis to predict the clinical response to imatinib in patients with advanced gastrointestinal stromal tumours entered on phase I and II studies of the EORTC Soft Tissue and Bone Sarcoma Group. European Journal of Cancer 2004; 40: 68995.

50. Koswadakis N, Visvardis EE, Kartsaklis P, Tsimara M, Chatziantoniou A, Panopoulos I ET aL. The role of surgery in the management of gastrointestinal stromal tumors(GIST) in the era of imatinib mesylate effectiveness. Surgical Oncology 2005; 14: 75-84.

51. Cheu L, Trent J, Wu E, Fuluer G, Ramdas L, Zhang W ET AL. A missense mutation in kit kinase domain 1 correlates with imatinib resistance in gastrointestinal stromal tumors occurs through secondary gene mutation. Clin Cancer Res 2005; 11: 4182-90.

52. Roch E, Goodman U, Jiang J, Malyoob K, Verbois L, Morse D ET AL. Food and Drugs Administration Drug Approval Summary: Sunitinib maleate for the treatment of gastrointestinal stromal tumor and advan- 
ced renal cell carcinoma. The Oncologist 2007; 12: 107-13.

53. Aparicio T, Boige V, Sabourin JC, Cremu P, Ducreux M, Le Cesue A et al Prognostic factors afther surgery of primary resectable gastrointestinal stromal tumours. EJSO 2004; 30: 1098-103.

54. Perez E, Livingstone S, Franceschi D, Roche Lima C, LeE D, Hodgson N et aL. Current incidence and outcomes of gastrointestinal mesenchymal tumors including gastrointestinal stromal tumors. J Am Coll Sur 2006; 202: 623-9.
55. Scaife CL, Hunt KK, Patel SR, Benjamin RS, Burgess MA, CHEN LL ET AL. Is there a role for surgery in patients with unresectable c c-kit (+) gastrointestinal stromal tumours treated with imatinib mesylate? Am J Surg 2003; 186: 665-9.

56. Joensuu H, Fletcher Ch, Dimitrijevic S, Silberman S, RoberTs P, Demetri G. Management of malignant gastrointestinal stromal tumours. Lancet Oncol 2002; 3: 655-64.

57. Bórquez P. Tumores del estroma gastrointestinal (GIST). Carta al editor. Rev Méd Chile 2006; 134: 1947. 